\title{
INVESTIGATING ACOUSTIC CHARACTERISTICS OF SOUND-ABSORBING MATERIALS BY THE THREE-MICROPHONE METHOD
}

\author{
Gleb Nazarov * and Alexandr Komkin \\ B M STU, Power Engineering Department, 105005, M oscow, Russia
}

\begin{abstract}
The paper reviews the existing methods for measuring the acoustic characteristics of sound-absorbing materials. The description of the used test bench for measuring the acoustic characteristics of materials by the three-microphone method is given. The results of the test measurements of the fibrous sound-absorbing material characteristics carried out at this test bench are presented. The analysis of the obtained results is carried out.
\end{abstract}

\section{Introduction}

Noise pollution is one of the main environmental problems of modern civilization. The only way of solving this problem is to use noise reduction devices, which often contain soundabsorbing materials (SAM). Such devices include, for example, dissipative noise suppressors. To increase the efficiency of such devices, it is necessary to know the acoustic characteristics of the sound-absorbing materials used. These characteristics can be divided into two groups. The first group includes the acoustic characteristics of a material sample that has a certain size. The acoustic characteristics of the sample include: the reflection coefficient $\mathrm{R}$, determined by the ratio of the amplitudes of the sound waves reflected from the sample and incident on it; absorption coefficient $\alpha=1-|R|^{2}$, as well as dimensionless acoustic impedance $Z_{s}=(1+R) /(1-R)$. The second group of characteristics includes the characteristics of the SAM itself. The acoustic characteristics of the material include wave impedance $Z_{m}$ and propagation constant $\mathrm{km}$ [1]. Acoustic impedance and complex wavenumber are fundamental acoustic properties of materials.

In accordance with two groups of acoustic characteristics, two groups of measurement methods can be distinguished: methods that allow to measure the acoustic characteristics of an SAM sample and methods that allow measuring the characteristics of the SAM itself.

As a rule, the measurements of the acoustic characteristics of the SAM samples are carried out in an interferometer. An overview of methods for measuring such characteristics is given in [2]. Previously, the main method of this kind of measurements was the standing wave method. In this case, in an impedance tube (Kundt's tube), with a speaker at one end of the tube and a test sample at the other, a standing wave is created due to the reflection of a harmonic sound wave created by the speaker from the sample. The acoustic characteristics of the sample are determined on the basis of measurements with a probing microphone as a ratio

* Corresponding author: author@email.org 
be-tween the maximum and minimum sound pressure in the standing wave. The main disadvantage of this method is its laboriousness, since it is necessary to find the peaks for each frequency separately and do it manually.

Currently, the main method for measuring the characteristics of a sample is the transfer function method (two-microphone method) [3-5]. This method is pretty less laborious, since it allows you to find all the necessary parameters in one measurement; however, requires calibration. In this case, harmonic, random noise or a chirp signal can be used as a signal supplied to the speaker $[6,7]$.

The acoustic characteristics of the SAM itself can be obtained by modifying the methods for sample characteristics measurement in two ways: either by performing additional measurements when changing the location of the sample or its geometric characteristics (these are the two-thickness and two-cavity methods); or using additional microphones in the measuring system [8].

The broadband two-cavity method was implemented in 1989 [9]. The acoustic characteristics of the material are determined from two measurements of the surface acoustic impedance with two different air cavities behind the material sample. This method is essentially a two-step use of the transfer-function method to measure surface impedance. However, for materials with high scattering power, the method may not be accurate.

Adding one or more additional measuring devices behind the sample allows you to find the acoustic characteristics of the material. It is believed that the more additional microphones are used, the more accuracy is achieved [10]. The most widely used methods are using four and three microphones.

The four-microphone method was proposed in [11]. The essence of the four-microphone method is to add two microphones to the additional cavity of the interferometer behind the sample. Further, from the readings of four microphones, a transmission matrix for the sample is found, which values allow to find the acoustic characteristics [11-14]. The dis-advantages of this method include a rather large amount of computation.

Along with this, the three-microphone method proposed in [15] finds great application. In this study, a sample of the tested material is installed on a rigid wall, two microphones are standardly placed on the wall of the impedance tube, and the third microphone is installed flush-face with the front surface of the sample, which creates certain difficulties. Therefore, the more convenient modification of this method for implementation was proposed [16, 17], where the third microphone is installed flush-face with a rigid wall behind the sample of the examined material. The study [16] also includes comparative results of measuring the characteristics of the SAM by three different methods, which showed large errors in the range below $500 \mathrm{~Hz}$ and good convergence at higher frequencies. In this article, this method will be considered.

\section{Three-microphone method}

The three-microphone measurement scheme is shown in Figure 1. This method allows to obtain both the acoustic characteristics of a material sample and the acoustic characteristics of the material itself. 


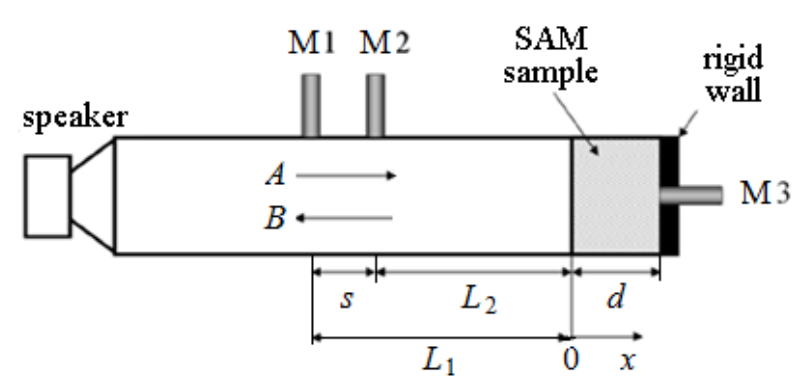

Fig. 1. Three-microphone measurement scheme.

Sound pressure in impedance tube:

$$
P(x)=A e^{-i k_{0} x}+B e^{i k_{0} x}
$$

where A and B are amplitudes of forward and backward waves.

The amplitudes of sound pressure in the impedance tube at measurement points 1 and 2 with coordinates $x_{1}=-L_{1}$ and $x_{2}=-L_{2}$ :

$$
\begin{aligned}
& P_{\mathrm{M} 1}=A e^{i k_{0} L_{1}}+B e^{-i k_{0} L_{1}} ; \\
& P_{\mathrm{M} 2}=A e^{i k_{0} L_{2}}+B e^{-i k_{0} L_{2}} .
\end{aligned}
$$

The solution to system (2) gives the following expressions for the amplitudes:

$$
\begin{aligned}
& A=P_{\mathrm{M} 2} \frac{H_{12}-e^{-i k_{0} s}}{2 i \sin k_{0} s} e^{-i k_{0} L_{2}} \\
& B=P_{\mathrm{M} 2} \frac{e^{i k_{0} s}-H_{12}}{2 i \sin k_{0} s} e^{i k_{0} L_{2}},
\end{aligned}
$$

where $H_{12}=P_{\mathrm{M} 1} / P_{\mathrm{M} 2} ; s=L_{1}-L_{2}$.

This allows to obtain expressions for the reflection coefficient at the front wall of the sample:

$$
R=\frac{B}{A}=\frac{e^{i k_{0} s}-H_{12}}{H_{12}-e^{-i k_{0} s}} e^{i 2 k_{0} L_{2}}
$$

and the amplitude of the sound pressure at this wall:

$$
P(0)=A+B=A(1+R)
$$

Considering that according to (2)

$$
A=P_{\mathrm{M} 2} \frac{1}{e^{i k_{0} L_{2}}+R e^{-i k_{0} L_{2}}},
$$

we rearrange (6) in the form: 


$$
P(0)=P_{\mathrm{M} 2} \frac{1+R}{e^{i k_{0} L_{2}}+R e^{-i k_{0} L_{2}}} .
$$

Let us now proceed to consider the propagation of sound in the sample. The sample is characterized by a propagation constant $\mathrm{km}$ and wave impedance $Z_{m}$, which for the test sample of sound-absorbing material are complex quantities. The amplitude of the sound wave in the sample, by analogy with (1), is written in the form:

$$
P_{2}(x)=A_{2} e^{-i k_{m} x}+B_{2} e^{i k_{m} x}
$$

where $A_{2}$ and $B_{2}$ are amplitudes of forward and backward waves.

At the front wall of the sample, at $x=0$, it is obvious:

$$
P_{2}(0)=A_{2}+B_{2}=P(0)
$$

At the rear wall of the sample $(x=d)$, the sound pressure amplitude can be found by the formula:

$$
P_{2}(d)=A_{2} e^{-i k_{m} d}+B_{2} e^{i k_{m} d}
$$

This value is measured by the third microphone, so that $P_{2}(d)=P_{\mathrm{M} 3}$. Relationship between amplitudes $A_{2}$ and $B_{2}$ depends on the coefficient of reflection from the rigid wall $R_{3}$, which in turn is determined by the formula:

$$
R_{3}=\frac{B_{2} e^{i k_{m} d}}{A_{2} e^{-i k_{m} d}}=\frac{Z_{3}-Z_{m}}{Z_{3}+Z_{m}},
$$

where $Z_{3}$ is the impedance of the rigid wall.

It is usually assumed that the surface flush with which the third microphone is installed is absolutely rigid, for which $Z_{3}=\infty$. Then, as follows from (10), $R_{3}=1$, which means that the amplitudes of the incident and reflected waves at this surface should be equal. This condition can be written as:

$$
A_{2} e^{-i k_{2} d}=B_{2} e^{i k_{2} d}=P_{\mathrm{M} 3} / 2
$$

Then, considering (10):

$$
\left(e^{i k_{m} d}+e^{-i k_{m} d}\right) P_{\mathrm{M} 3} / 2=P(0)
$$

or

$$
\cos k_{m} d=P(0) / P_{\mathrm{M} 3}
$$

From that, considering (8), the required expression for the wave number $\mathrm{km}$ is obtained:

$$
k_{m}=\frac{1}{d} \arccos \left(P(0) / P_{\mathrm{M} 3}\right)=\frac{1}{d} \arccos \left(\frac{1+R}{e^{i k_{0} L_{2}}+R e^{-i k_{0} L_{2}}} H_{23}\right)
$$


where $H_{23}=P_{\mathrm{M} 2} / P_{\mathrm{M} 3}$.

Let us now find an expression for the wave impedance $Z_{m}$. It can be expressed through the surface (input) impedance of the sample $Z_{s}-$ dimensionless (normalized to the air impedance $Z_{0}$ ) value, which is determined by the formula (17):

$$
Z_{S}=\frac{1+R}{1-R}
$$

In this case, the relation takes place:

$$
Z_{S}=Z_{m} \frac{Z_{3}+i Z_{m} \operatorname{tg} k_{m} d}{Z_{m}+i Z_{3} \operatorname{tg} k_{m} d}
$$

Further in this formula, as in the derivation of formula (14), it is assumed that $Z_{3}=\infty$. In this case, $(18)$ is reduced to the form

$$
Z_{m}=i Z_{S} \operatorname{tg} k_{m} d
$$

From this, considering (17) and (5), the required expression for the wave impedance of the sample is obtained:

$$
Z_{m}=\frac{H_{12} \sin \left(k_{0} L_{2}\right)-\sin \left(k_{0}\left(L_{2}+s\right)\right)}{H_{12} \cos \left(k_{0} L_{2}\right)-\cos \left(k_{0}\left(L_{2}+s\right)\right)} \operatorname{tg}\left(k_{m} d\right)
$$

Thus, the obtained formulas (16) and (20) make it possible to determine the acoustic characteristics of the sound-absorbing material from the measurement results. At the same time, we emphasize that these formulas were derived under the assumption that the wall in front of the sound-absorbing material, into which the third microphone is mounted, is absolutely rigid.

\section{Experimental setup}

The external view of the experimental setup developed in the «Technical acoustics» laboratory of BMSTU [18] is shown in Figure 2.

During measurements, white noise was used as the generated signal. Signals from measuring microphones were processed by a signal analyser using RT Pro Photon software. As a result, the transfer functions $H_{21}(f)$ and $H_{32}(f)$ were found between the measuring microphones. In this case, to reduce the errors of spectral estimates, we used the mode of linear averaging of the obtained transfer functions over a long-time interval. Further calculations to obtain acoustic characteristics of the materials under study were carried out on a personal computer in the Mathcad environment. 


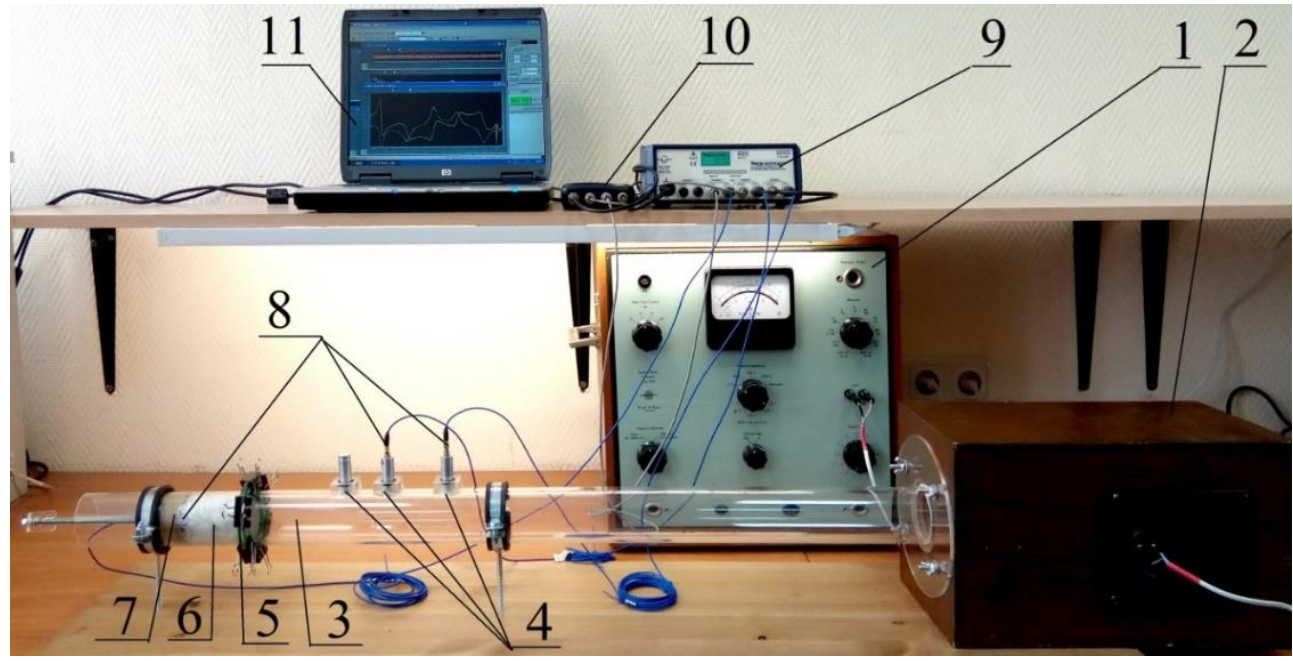

Fig. 2. General view of the experimental setup: 1 - B\&K 1402 white noise generator; 2 - speaker; 3 impedance plexiglass tube with an inner diameter of $54 \mathrm{~mm}$ and an outer diameter of $60 \mathrm{~mm} ; 4$ - three sockets for installing microphones, with a distance of 50 and $70 \mathrm{~mm} ; 5$ - sealed joint with a separating mesh between two pipes $740 \mathrm{~mm}$ and $200 \mathrm{~mm}$ long; 6 - sample of the investigated SAM; 7 - movable steel piston with an axisymmetric hole for the microphone; 8 - three PCB 378C10 measuring condenser microphones; 9 - PCB 482C05 four-channel preamplifier of microphone signals; 10 - B\&K PHOTON+ signal analyser; 11 - personal computer..

The presented calculation results were limited to the frequency range $150 \mathrm{~Hz}-2.5 \mathrm{kHz}$. The lower limit of this range was due to the low coherence of signals at lower frequencies, which led to very large errors in the measurement results. The upper limit of the range is provided that only plane sound waves can propagate in the pipe. This condition has the form $f_{w}<f_{c r}$. Frequency $f_{c r}$ is determined by the inner pipe diameter $d_{i n}: f_{c r}=0,586 c_{0} / d_{i n}$. Hence, with the inner diameter of the impedance pipe $d_{i n}=54 \mathrm{~mm}$, we find $f_{c r}=3700 \mathrm{~Hz}$.

Calibration is an important preliminary step in ensuring reliable measurement results. In this regard, at the first stage, the reflection coefficient for a rigid wall of a piston was measured. The theoretical value of this coefficient should be equal to 1 . Correct calibration should bring the measurement results closer to this value. The dependences of the modulus of the reflection coefficient on frequency obtained from the results of the calibration for a rigid wall of a piston are shown in Figure 3.

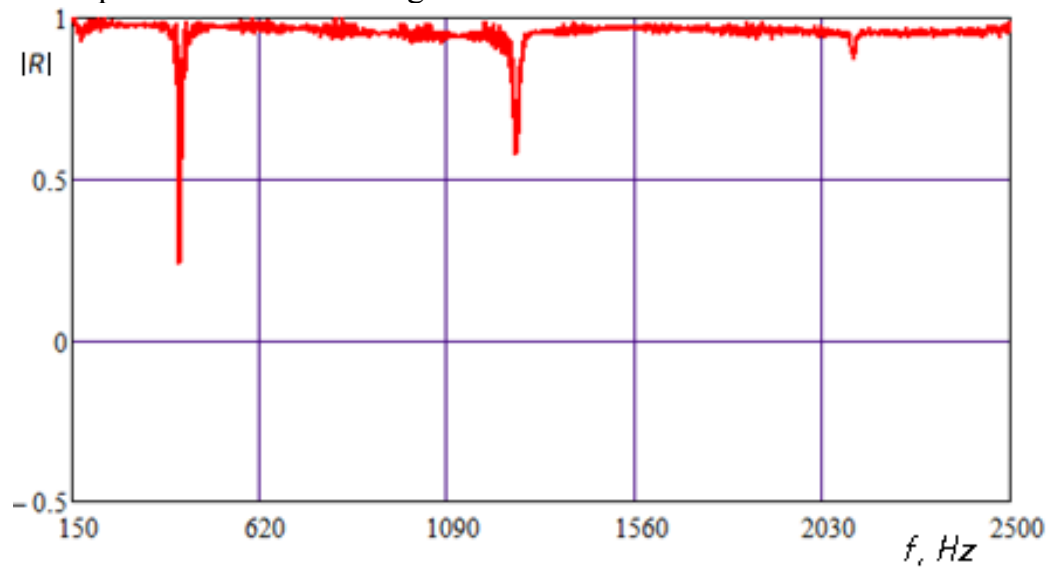

Fig. 3. Reflection coefficient modulus for a rigid wall. 
Changes in the modulus of the reflection coefficient in most of the considered frequency range are insignificant, with the exception of the dips at two frequencies associated with low coherence of the processed signals at these frequencies. In this case, the average value of the modulus of the reflection coefficient is $|R|_{a v}=0.969$.

\section{Measurement results}

Measurements of the acoustic characteristics of the SAM were carried out, which was mineral wool. A sample of material $58 \mathrm{~mm}$ thick and with a bulk density of $100 \mathrm{~kg} / \mathrm{m}^{3}$ was investigated. A sample of the investigated material is shown in Figure 4.

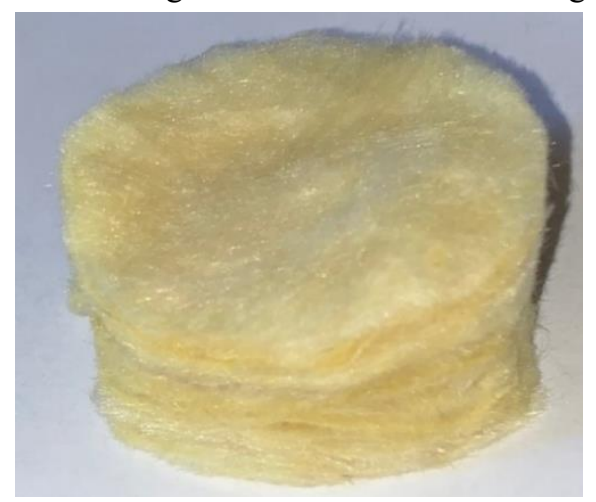

Fig. 4. Sample of the investigated SAM.

The frequency dependences of the reflection and absorption coefficients obtained for this sample of the SAM are shown in Figure 5. As expected, in the low-frequency range with increasing frequency, the curve of the modulus of the reflection coefficient decreases monotonically. The curve corresponding to the absorption coefficient is a specular reflection of the curve of the reflection coefficient modulus, reaching a maximum value close to 1 .

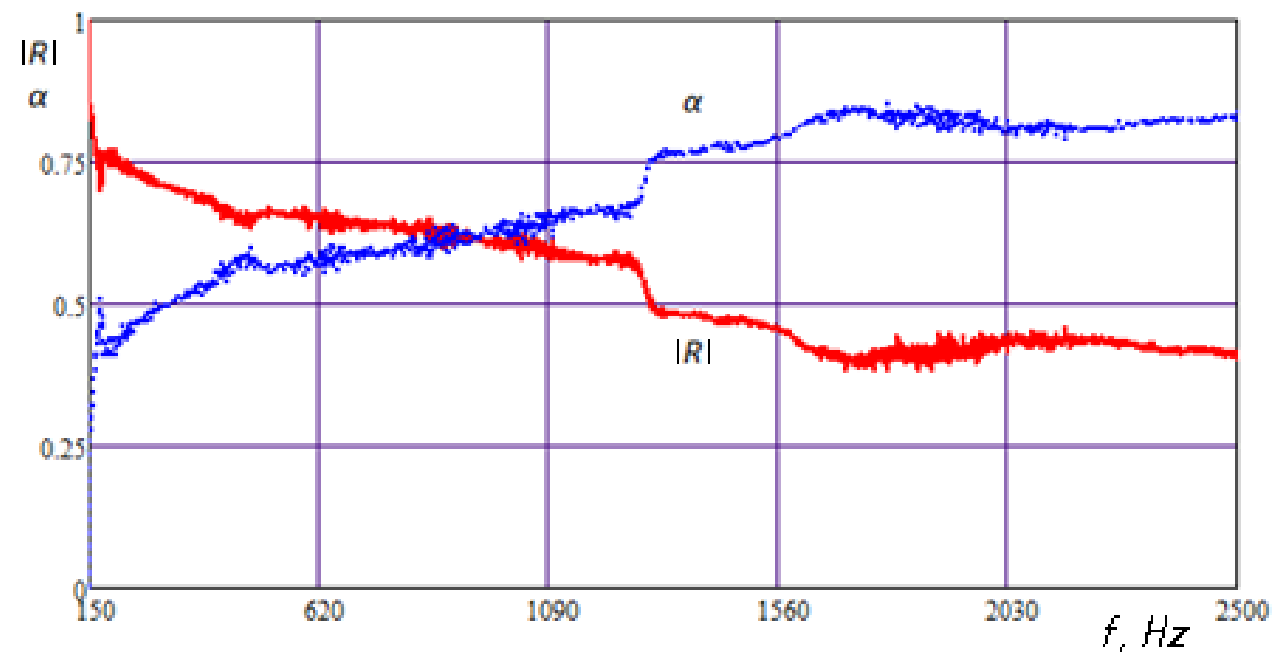

Fig. 5. Acoustic characteristics of the investigated SAM sample: $(-)$ - the modulus of the reflection coefficient; $(\cdots)$ - the modulus of the absorption co-efficient. 
Further, in the Mathcad software environment, the propagation constant km and the wave impedance $Z_{m}$ were calculated for the considered SAM sample, based on the obtained measurement results using the above formulas (16) and (20).

In addition, the values obtained by the experimental method can be calculated using the empirical Delany-Bazley formulas [19], which in the general case can be represented in the form [20]:

$$
\begin{aligned}
& k_{m} / k_{0}=1+a_{2}\left(\frac{\rho_{0} f}{r}\right)^{b_{2}}-i a_{1}\left(\frac{\rho_{0} f}{r}\right)^{b_{1}} \\
& Z_{m} / Z_{0}=1+a_{4}\left(\frac{\rho_{0} f}{r}\right)^{b_{4}}-i a_{3}\left(\frac{\rho_{0} f}{r}\right)^{b_{3}}
\end{aligned}
$$

where $k_{0}$ and $Z_{0}$ are the wavenumber and wave resistance for air, respectively; $r$ is the blowout resistance of the SAM; $a_{1}, a_{2}, a_{3}, a_{4}, b_{1}, b_{2}, b_{3}, b_{4}$ are some constants.

Blowout resistance $r$ for the investigated fiberglass-based SAM can be calculated from the empirical dependence of the blowout resistance of the material on its bulk density $\rho_{m}$, determined by the formula [21] $r=1,51 \rho_{m}^{2}$. Hence, we find that a sample of material with a bulk density of $100 \mathrm{~kg} / \mathrm{m}^{3}$ used during measurements will have a blowout resistance of $r=1,51 \cdot 10^{4} \mathrm{~N} \cdot \mathrm{s} / \mathrm{m}$.

In Figure 6 solid lines represent the dependences of the real and imaginary parts of the propagation constant on frequency obtained from the experimental results. The dotted lines in this figure show the dependences calculated by the empirical formula (21). For the coefficients of approximation in this dependence, the following values were taken: $a_{1}=$ $0,529, a_{2}=0,274, b_{1}=-0,595, b_{2}=-0,700$.

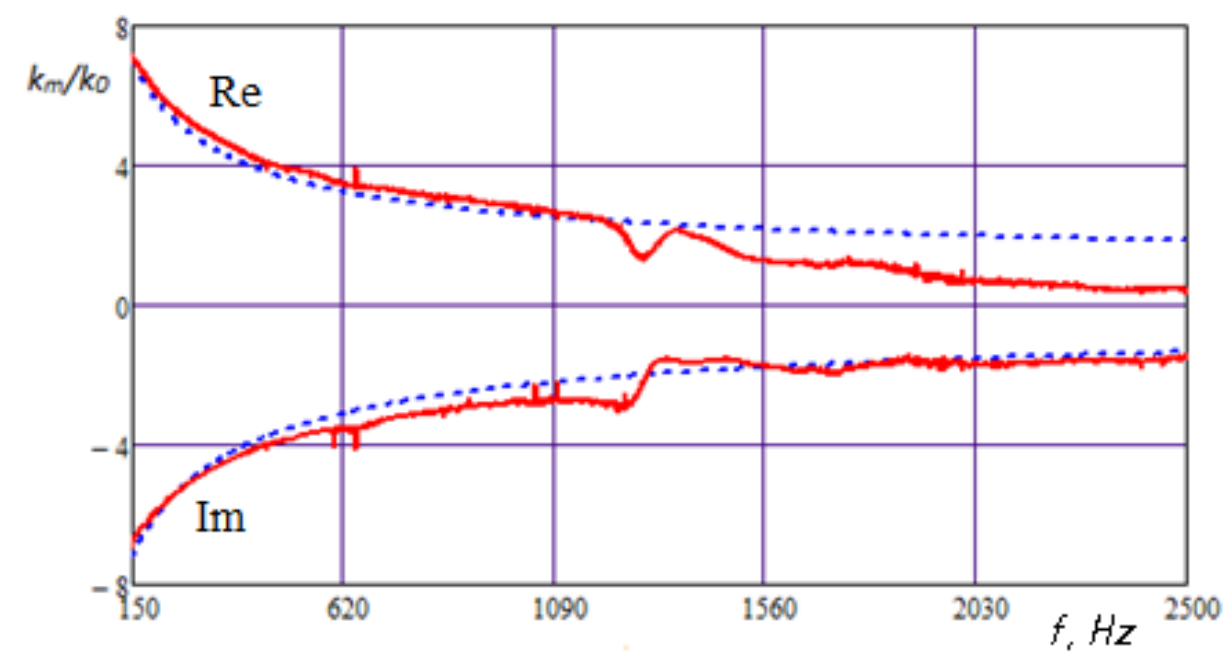

Fig. 6. Dependence of the dimensionless propagation constant of the SAM on the frequency: (- ) experiment; $(\ldots \ldots)$ - empirical dependencies.

Similarly, Figure 7 shows the dependences of the real and imaginary parts of the dimensionless wave impedance of the investigated material on frequency, obtained from the experimental results and approximated by the empirical dependence (22). For the coefficients of approximation in this dependence, the following values were taken: $a_{3}=0,261, \mathrm{a}_{4}=0,171$, $b_{3}=-0,650, b_{4}=-0,850$. 


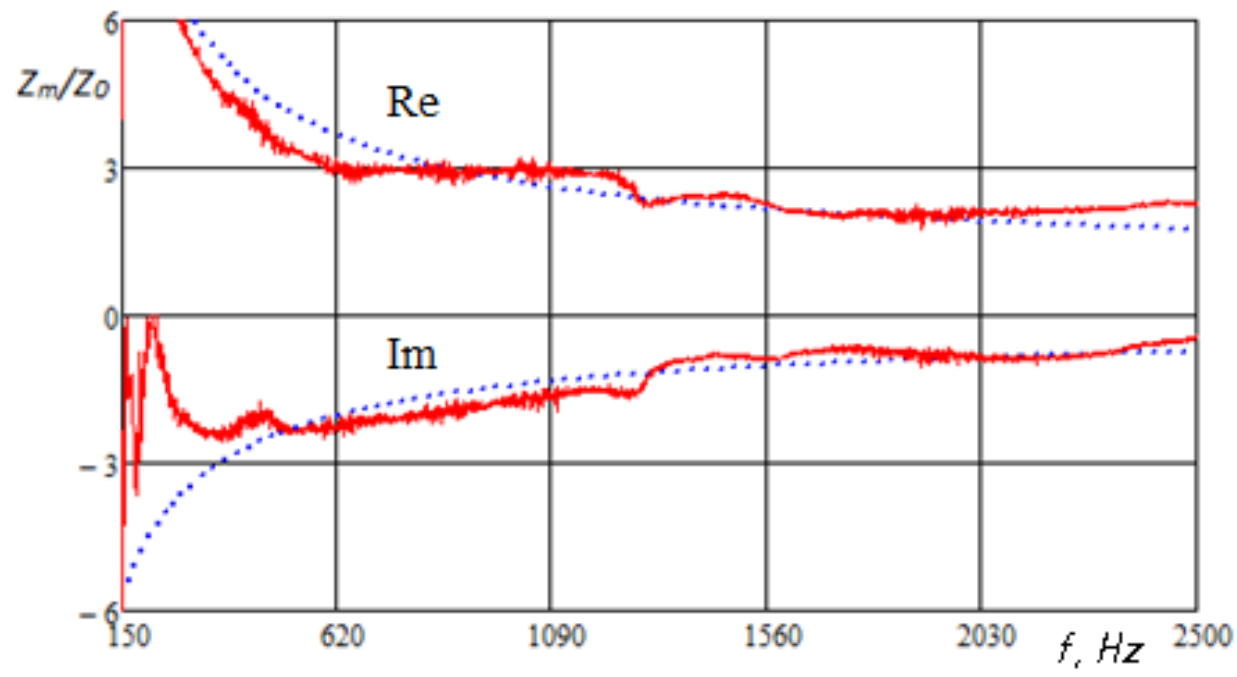

Fig. 7. Dependence of the normalized acoustic impedance of the SAM on the frequency: ( - experiment; $(\ldots$.$) - empirical dependencies.$

The results obtained generally agree with the results of some other studies $[11,14,16]$, which show that the reliability of the results obtained should be limited to the frequency range above $500 \mathrm{~Hz}$. And although it was shown in [10] that with decreasing frequency, measurement errors increase significantly, the reasons for this phenomenon require further more detailed investigation.

\section{Conclusion}

The review of methods for measuring the acoustic characteristics of sound-absorbing materials made it possible to establish the expediency of using the three-microphone method for this purpose. The used experimental setup, which implements this measurement method, has shown its efficiency. The acoustic characteristics of the investigated SAM based on mineral wool have been determined. The obtained experimental results are approximated by empirical dependences.

\section{References}

1. C. Zwikker, C. W. K osten, Sound absorbing materials (Elsevier, NY -L ondon, 1949)

2. A. I. Komkin, M easur. Techn. 43 (3), 290 (2003)

3. J. Y. Chung, D. A. Blaser, J. A coust. Soc. A m. 68 (3), 907 (1980)

4. J. Y. Chung, D. A. Blaser, J. A coust. Soc. A m. 68 (3), 914 (1980)

5. ISO 10534-2: A coustics. D etermination of sound absorption coefficient and impedance in impedance tubes. Transfer-function method (2001)

6. J. K unio, T. Y oo, K. Hou, J. S. Bolton, J. Enok, A Comparison of Two and Four Microphone Standing Wave Tube Procedures for Estimating the Normal Incidence Absorption Coefficient, in Proceeding of the INTER-N OISE 2009, A ugust 23-26 2009, Ottawa, Canada (2009)

7. J. D. M clntosh, M. T. Zuroski, R. F. L ambert, J. A coust. Soc. A m. 88 (4), 1929 (1990) 
8. A . I. Komkin, N. A . Nikiforov, B ezopasnost@ehiznedeyatel @osti, 22 (2006) [in Russsian]

9. H. Utsuno, T. Tanaka, T. Fujikawa, A. F. Seybert, J. A coust. Soc. Am. 86 (2), 637 (1989)

10. R. T. M uehleisen, C.W. Beamer IV , A RLO, 3 (4),112 (2002)

11. B. H. Song, J. S. Bolton, J. A coust. Soc. A m. 107 (3), 1131 (2000)

12. R. T. M uehleisen, C.W. B eamer IV, B. D. Tinianov, J. A coust. Soc. A m. 117 (2), 536 (2005)

13. A. I. Komkin, S. I. Y udin, Measurement and calculation of acoustic characteristics of fibrous sound-absorbing materials, in Proceedings of the XIX session of the R ussian acoustic society, 24-28 september 2007, M oscow, Russia (2007) [in Russsian]

14. S. Liang et al., J. A coust. Soc. A m. 126 (6), 3049 (2009)

15. T. I wase, Y. Izumi, and R. K awabata, A new measuring method for sound propagation constant by using sound tube without any air spaces back of a test material, in Proceedings of INTER-N OISE 98, 16-18 November 1998, Christchurch, N ew Zealand, (1998).

16. Y. Salissoua, R. Panneton, J. A coust. Soc. A m. 107 (3), 2868 (2009)

17. O. Doutres, Y. Salissou, N. A talla, R. Panneton, A ppl. A coust. 71 (6), 506 (2010)

18. I. A. Ionov, N. S. N esterov, A .I. K omkin, Development of a stand for the study of acoustic characteristics of sound-absorbing materials, in Proceedings of the II Conference of $Y$ oung Scientists and Specialists on Environmental A coustics, ASO2017, 19 M ay 2017, M oscow, Russia (2017) [in Russsian]

19. M. E. Delany, E. N. Bazley, A pplied A coustics, 1970, 3, 105 (1970)

20. I. A. Ionov, A I. K omkin, Empirical formulas for describing the acoustic characteristics of sound-absorbing materials. Overview, in Proceedings of the III Conference of $Y$ oung Scientists and Specialists on Environmental A coustics, A SO2018, 18 M ay 2018, M oscow, Russia (2018) [in Russsian]

21. A . I. Komkin, V. A . Lvov, N. S. Nesterov, M easur. Techn. 57, (7), 62 (2017) 